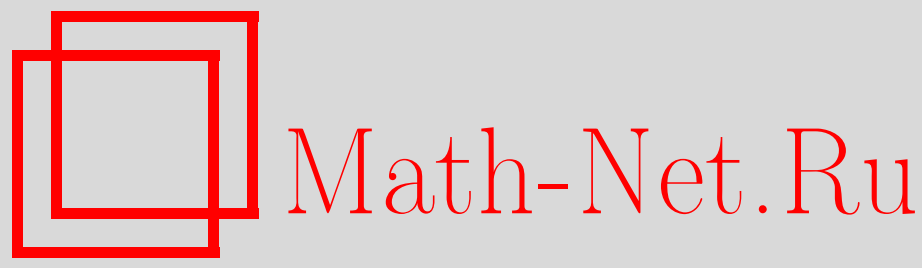

В. Г. Саргсян, Асимптотика логарифма числа множеств, $(k, l)$-свободных от сумм, в абелевой группе, Дискрет. матем., 2014, том 26, выпуск 4, 91-99

DOI: https://doi.org/10.4213/dm1307

Использование Общероссийского математического портала Math-Net.Ru подразумевает, что вы прочитали и согласны с пользовательским соглашением http://www .mathnet.ru/rus/agreement

Параметры загрузки:

IP : 54.166 .219 .16

26 апреля 2023 г., 14:37:21

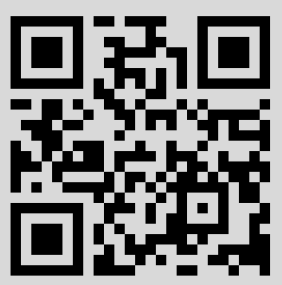




\title{
Асимптотика логарифма числа множеств, $(k, l)$-свободных от сумм, в абелевой группе
}

\author{
() 2014 г. В. Г. Саргсян*
}

Подмножество $A$ элементов группы $G$ называется $(k, l)$-свободным от сумм, если уравнение $x_{1}+\ldots+x_{k}=y_{1}+\ldots+y_{l}$ не имеет решений в множестве $A$. Получена асимптотика логарифма числа множеств, $(k, l)$-свободных от сумм, в абелевой группе.

Работа выполнена при финансовой поддержке РФФИ, проект № 13-01-00958a.

Ключевые слова: множество, свободное от сумм, характеристическая функция, группа, прогрессия, смежный класс.

\section{1. Введение}

Пусть $G$ - множество с определенной на нем операцией сложения, а $k$ и $l$ - неотрицательные целые числа, удовлетворяющие условию $k+l \geqslant 3$. Подмножество $A \subseteq G$ называется $(k, l)$-свободным от сумм $((k, l)$-МСC), если уравнение

$$
x_{1}+\ldots+x_{k}=y_{1}+\ldots+y_{l}
$$

не имеет решений в множестве $A$. Семейство всех $(k, l)$-MCC в $G$ обозначим через $S F_{k, l}(G)$. Множество, $(2,1)$-свободное от сумм, называется просто множеством, свободным от сумм (МСC). Для натуральных чисел $m$ и $n$ обозначим через $[m, n]$ множество таких натуральных чисел $x$, что $m \leqslant x \leqslant n$.

В 1988 г. П. Камерон (Р. Cameron) и П. Эрдёш (Р. Erdös) [1] предположили, что $S F_{2,1}([1, n])=O\left(2^{n / 2}\right)$. В частности, они доказали, что существуют такие константы $c_{0}$ и $c_{1}$, что $\left|S F_{2,1}([[n / 3], n])\right| \sim c_{0} 2^{n / 2}$ для четных $n$, и $\left|S F_{2,1}([[n / 3], n])\right| \sim c_{1} 2^{n / 2}$ для нечетных $n$.

Н. Калкин (N. Calkin) [2] и, независимо, Н. Алон (N. Alon) [3] доказали, что $\limsup \frac{2}{n} \log \left|S F_{2,1}([1, n])\right| \leqslant 1$. (Здесь и далее $\log x=\log _{2} x$.)

Доказательство гипотезы Камерона-Эрдёша и асимптотика числа МСС в отрезке $[1, n]$ были найдены А. А. Сапоженко [4] и, независимо, Б. Грином (B. Green) [5]. Доказано, что $\left|S F_{2,1}([1, n])\right| \sim c(n) 2^{n / 2}$, где константа $c(n)$ зависит от четности $n$.

В 1991 г. Н. Алон [3] доказал, что для любого $\varepsilon>0$ число МСС произвольной конечной группы не превосходит $2^{n / 2+\varepsilon n}$ при всех достаточно больших $n$. Далее данный результат уточнялся для разных подклассов конечных абелевых групп. Так, в 2002 г. А.А. Сапоженко [6] и, независимо, Лев, Лучак и Шон (V. Lev, T. Luczak, T.

* Место работы: МГУ имени М.В. Ломоносова, e-mail: vahe_sargsyan@ymail.com 
Schoen) [7] получили асимптотику максимально возможного числа МСС для конечных абелевых групп, содержащих хотя бы одну подгруппу индекса 2. Далее группу вычетов по модулю $n$ обозначаем через $\mathbf{Z}_{n}$.

В 2002 г. В. Лев и Т. Шон [8] доказали, что если $p$ - достаточно большое простое число, то справедливы оценки

$$
2^{\lfloor(p-2) / 3\rfloor}(p-1)\left(1+O\left(2^{-\varepsilon_{1} p}\right)\right) \leqslant\left|S F_{2,1}\left(\mathbf{Z}_{p}\right)\right| \leqslant 2^{p / 2-\varepsilon_{2} p},
$$

где $\varepsilon_{1}$ и $\varepsilon_{2}-$ положительные константы.

В 2005 г. Б. Грин и И. Ружа (I. Ruzsa) [9] с использованием преобразований Фурье получили асимптотику логарифма числа МСС в конечных абелевых группах. Они доказали, что для любой конечной абелевой группы $G$ справедливо соотношение $\log \left|S F_{2,1}(G)\right| \sim \mu_{2,1}(G)$, где $\mu_{2,1}(G)$ - максимальная мощность МСС в $G$.

В 2009 г. А. А. Сапоженко [10] получил асимптотику числа МСС в группах простого порядка.

Теорема 1. Для любого $\alpha \in\{-1,1\}$ существует такая константа $c_{\alpha}$, что для любого $\varepsilon>0$ существует такое натуральное число $N$, что для любого простого $p$ вида $p \equiv \alpha(\bmod 3), p>N$, выполняются неравенства

$$
1 \leqslant \frac{\left|S F_{2,1}\left(\mathbf{Z}_{p}\right)\right|}{c_{\alpha}(p-1) 2^{\lfloor(p-2) / 3\rfloor}}<1+\varepsilon .
$$

Вместе с тем интенсивно рассматривались обобщения проблемы Камерона-Эрдёша. В частности, речь шла о числе $(k, l)$-МСС.

В 1996 г. Н. Калкин и А. Тейлор (А. Taylor) [11] доказали, что существует такая константа $C_{k}, k \geqslant 3$, что $\left|S F_{k, 1}([1, n])\right|$ не превосходит $C_{k} 2^{(k-1) n / k}$. В 1998 г. Ю. Билу (Yu. Bilu) [12] доказал, что $\left|S F_{l+1, l}([1, n])\right|=(1+\bar{o}(1)) 2^{\lfloor(n+1) / 2\rfloor)}$, a Н. Калкин и Дж. Томсон (J. Thomson) [13] доказали, что существует такая константа $C_{k, l}$, $k \geqslant 4 l-1$, что $S F_{k, l}([1, n])$ не превосходит $C_{k, l} 2^{(k-l) n / k}$.

В 2000 г. Т. Шон [14] установил асимптотику числа $(k, l)$-MCС в отрезке натуральных чисел $[1, n]$ при некоторых ограничениях на $k$ и $l$. В 2003 г. В. Лев [16] получил верхную оценку числа $(k, l)$-МСС в отрезке натуральных чисел $[1, n]$.

В 2012 г. В.Г. Саргсян [15] получил асимптотику логарифма числа $(k, l)$-МСС в группе простого порядка.

В 2007 году Б. Байнок (B. Bajnok) [18] получил верхнюю и нижнюю оценки максимальной мощности $(k, l)-\mathrm{MCC}$ в абелевой группе. Обозначим максимальную мощность $(k, l)$-МСС в $G$ через $\mu_{k, l}(G)$.

Теорема 2. Пусть $G$ - абелева группа порядка $n, a k$ u $l$ - различные положительные целье числа. Тогда справедливы неравенства

$$
\max _{d \mid \nu}\left\{\left(\left\lfloor\frac{d-1-\delta_{k, l}(d)}{k+l}\right\rfloor+1\right) \frac{n}{d}\right\} \leqslant \mu_{k, l}(G) \leqslant \max _{d \mid n}\left\{\left(\left\lfloor\frac{d-2}{k+l}\right\rfloor+1\right) \frac{n}{d}\right\},
$$

где $\nu$ - экспонента группъ $G, a \delta_{k, l}(d)=$ НОД $(d, k-l)$.

В 2013 году В.Г. Саргсян [19] получил такие же верхние и нижние оценки для максимальной мощности $(k, 0)-\mathrm{MCC}$ в абелевой группе.

Теорема 3. Пусть $G$ - абелева группа порядка $n u k \geqslant 3$. Тогда справедливы неравенства

$$
\max _{d \mid \nu}\left\{\left(\left\lfloor\frac{d-1-\delta_{k}(d)}{k}\right\rfloor+1\right) \frac{n}{d}\right\} \leqslant \mu_{k, 0}(G) \leqslant \max _{d \mid n}\left\{\left(\left\lfloor\frac{d-2}{k}\right\rfloor+1\right) \frac{n}{d}\right\},
$$


где $\nu$ - экспонента группъ $G$, а $\delta_{k}(d)=$ НОД $(d, k)$.

В данной работе с использованием методов [9] получена асимптотика логарифма числа $(k, l)$-МСС для произвольных абелевых групп.

Теорема 4. Пусть $G$ - абелева группа порядка $n, a k$ a $l$ - неотрищательные целье числа, удовлетворяющие условию $k+l \geqslant 3$. Тогда для любого $\varepsilon>0$

$$
|\log | S F_{k, l}(G)\left|-\mu_{k, l}(G)\right|<\varepsilon n, \text { если п достаточно велико. }
$$

\section{2. Определения и вспомогательные утверждения}

Пусть $G$ - абелева группа порядка $n$ с операцией сложения. Характером группы $G$ называется такое отображение $\gamma: G \rightarrow \mathbb{C}$ такое, что для любых $x, y \in G$ имеют место равенства $|\gamma(x)|=1$ и $\gamma(x+y)=\gamma(x) \gamma(y)$. Обозначим через Г множество всех характеров группы $G$. Заметим, что $\Gamma$ образует группу с операцией $*$, а именно, $\gamma_{1} * \gamma_{2}(x)=\gamma_{1}(x) \gamma_{2}(x)$. Пусть $f: G \rightarrow \mathbb{R}$. Преобразованием Фуръе $f$ называется функция $\widehat{f}: G \rightarrow \mathbb{C}$, определяемая равенством $\widehat{f}(\gamma)=\sum_{x \in G} f(x) \gamma(x)$.

Лемма 1. Пусть $G$ - абелева группа порядка $n$ u $f: G \rightarrow \mathbb{R}$, a $\widehat{f}-$ преобразование Фуръе функиии $f$. Тогда при $k>l \geqslant 0$ справедливо равенство

$$
\sum_{\substack{x_{1}+\ldots+x_{k}=\\=y_{1}+\ldots+y_{l}}} f\left(x_{1}\right) \cdot \ldots \cdot f\left(x_{k}\right) \cdot f\left(y_{1}\right) \cdot \ldots \cdot f\left(y_{l}\right)=\frac{1}{n} \sum_{\gamma}(\widehat{f}(\gamma))^{k-l}|\widehat{f}(\gamma)|^{2 l} .
$$

Доказательство. С учетом того, что

$$
\sum_{\gamma \in \Gamma} \gamma(x)= \begin{cases}0, & \text { если } x \neq 0 \\ n, & \text { если } x=0\end{cases}
$$

имеем

$$
\begin{aligned}
& \sum_{\substack{x_{1}+\ldots+x_{k}=\\
=y_{1}+\ldots+y_{l}}} f\left(x_{1}\right) \cdot \ldots \cdot f\left(x_{k}\right) \cdot f\left(y_{1}\right) \cdot \ldots \cdot f\left(y_{l}\right)= \\
& =\frac{1}{n} \sum_{\substack{x_{i}, y_{j} \in G \\
i \in[1, k], j \in[1, l]}} \sum_{\gamma}\left(\gamma\left(x_{1}+\ldots+x_{k}-y_{1}-\ldots-y_{l}\right) \times\right. \\
& \left.\times f\left(x_{1}\right) \cdot \ldots \cdot f\left(x_{k}\right) \cdot f\left(y_{1}\right) \cdot \ldots \cdot f\left(y_{l}\right)\right)= \\
& =\frac{1}{n} \sum_{\gamma}\left(\left(\sum_{x_{1}} \gamma\left(x_{1}\right) f\left(x_{1}\right)\right) \cdot \ldots \cdot\left(\sum_{x_{k}} \gamma\left(x_{k}\right) f\left(x_{k}\right)\right) \times\right. \\
& \left.\times\left(\sum_{y_{1}} \gamma\left(-y_{1}\right) f\left(y_{1}\right)\right) \cdot \ldots \cdot\left(\sum_{y_{l}} \gamma\left(-y_{l}\right) f\left(y_{l}\right)\right)\right)= \\
& =\frac{1}{n} \sum_{\gamma}(\widehat{f}(\gamma))^{k}(\overline{\widehat{f}(\gamma)})^{l}=\frac{1}{n} \sum_{\gamma}(\widehat{f}(\gamma))^{k-l}|\widehat{f}(\gamma)|^{2 l} .
\end{aligned}
$$

Лемма 1 доказана. 
Для доказательства теоремы 4 используем метод гранулирования. Сущность этого метода состоит в том, что для оценки мощности множества $S F_{k, l}(G)$ строится такое семейство $\mathcal{F}$ «гранул» $F \in \mathcal{F}$, что каждый элемент множества $S F_{k, l}(G)$ содержится в некоторой грануле $F$ из построенного семейства $\mathcal{F}$, при этом $\log |\mathcal{F}|=\bar{o}(n)$ и в каждой грануле $F \in \mathcal{F}$ есть $\bar{o}\left(n^{k+l-1}\right)$ решений уравнения (1). Таким образом, строится семейство подмножеств группы $G$, обладающих следующими свойствами:

$-\log |\mathcal{F}|=\bar{o}(n)$

- для любого $A \in S F_{k, l}(G)$ существует такая гранула $F \in \mathcal{F}$, что $A \subseteq F$;

- в каждой грануле $F \in \mathcal{F}$ существует $\bar{o}\left(n^{k+l-1}\right)$ решений уравнения (1).

Будем рассматривать два типа гранул.

$L$-гранулой типа смежного класса называется объединение смежных классов группы $G$ по некоторой подгруппе порядка не меньше $L$.

Пусть $L$ - целое число и $d \in G$, причем $\operatorname{ord}(d) \geqslant L$, где $\operatorname{ord}(d)-$ порядок элемента $d$. Рассмотрим подгруппу $G$, порожденную элементом $d$, и разобъём каждый её смежный класс на $\lfloor\operatorname{ord}(d) / L\rfloor$ прогрессий вида $\{x+i d \mid 0 \leqslant i \leqslant L-1\}$ и одно «остаточное» множество мощности менее $L$. Для каждого $d \in G$ фиксируем одно такое разбиение. Объединение полученных прогрессий называется $L$-гранулой типа прогрессии.

Доказательства следующих двух лемм можно найти в работе [9].

Лемма 2. Пусть $G$ - абелева группа порядка $n$. Тогда при достаточно большом $n$ число L-гранул обоих типов (прогрессии и смежсного класса) в группе $G$ не превосходит $2^{3 n / L}$.

Лемма 3. Пусть $M-$ множество мощности $n$ u $n$ достаточно велико, а $\rho$ вещественное число, меньшее некоторой абсолютной положительной постоянной. Тогда число подмножеств множества $M$ мощности не более рп не превосходит $2^{n \sqrt{\rho}}$.

Естественно, что если в множестве «мало» решений уравнения 1, то в нем существует подмножество, свободное от решений уравнения 1, с «большой» мощностью. Доказательство этого факта можно найти в работе [17].

Теорема 5. Пусть $m \geqslant 3, A_{1}, \ldots, A_{m}-$ такие подмножества абелевой группь $G$ порядка $n$, что существует не более $\bar{o}\left(n^{m-1}\right)$ решений уравнения $x_{1}+\ldots+x_{m}=0$ при $x_{i} \in A_{i}, i=1, \ldots, m$. Тогда существуют такие подмножества $A_{1}^{\prime}, \ldots, A_{m}^{\prime}$, что $A_{i}^{\prime} \subseteq A_{i},\left|A_{i} \backslash A_{i}^{\prime}\right|=\bar{o}(n)$, и решений уравнения $x_{1}+\ldots+x_{m}=0$ при $x_{i} \in A_{i}^{\prime}$, $i=1, \ldots, n$, нет.

Далее характеристическую функцию множества $A$ обозначаем через $A(x)$.

\section{3. Гранулирование}

Сущность следующей леммы состоит в том, что для каждого $A \in S F_{k, l}(G)$ строится «подходящая» гранула.

Лемма 4 (Гранулирование).

Пусть $G$ - абелева группа порядка $n$ u $A \in S F_{k, l}(G)$, a $\varepsilon \in\left(0, \frac{1}{2}\right), L u L^{\prime}-$ положительные числа, удовлетворяющие неравенству

$$
n>L^{\prime}(4 L / \varepsilon)^{4^{2(k+l)+1}(k+l)^{2} \varepsilon^{-2(k+l+1)}} .
$$

Тогда существует такое подмножество $A^{\prime} \subseteq G$, что 
(i) $A^{\prime}$ - либо L-гранула типа прогрессии, либо $L^{\prime}$-гранула типа смежного клас$c a$;

(ii) $\left|A \backslash A^{\prime}\right| \leqslant \varepsilon n$;

(iii) $A^{\prime}$ содержит не более $\varepsilon n^{k+l-1}$ решений уравнения (1).

Доказательство. Положим $\delta=\varepsilon^{k+l+1} /(k+l) 4^{k+l}$. Сначала докажем, что существует подмножество $P \subseteq G$, удовлетворяющее условиям

(А) $P$ - либо прогрессия вида $\{d i \mid L-1 \leqslant i \leqslant L-1\}$, причем $\operatorname{ord}(d) \geqslant 2 L / \varepsilon$, либо подгруппа группы $G$ порядка не менее $L^{\prime}$;

(В) для любых $B \subseteq G$ и $\gamma \in \Gamma$ имеет место неравенство $|\widehat{B}(\gamma)(1-g(\gamma))| \leqslant \delta n$, где $g(\gamma)=|P|^{-1} \sum_{p \in P} \gamma(p)$.

Пусть $R$ - множество таких характеров $\gamma$, что $|\widehat{B}(\gamma)|>\delta n / 2$, а $\Gamma_{1}$ - подгруппа группы $\Gamma$, порожденная множеством $R$. Рассмотрим подгруппу $G_{1}$ группы $G$ :

$$
G_{1}=\left\{x \in G \mid \gamma(x)=1 \text { для любого } \gamma \in \Gamma_{1}\right\} \text {. }
$$

Рассмотрим два случая.

1) Пусть $\left|G_{1}\right| \geqslant L^{\prime}$. Положим $P=G_{1}$. Так как $g(\gamma) \in[-1,1]$ при $\gamma \in \Gamma \backslash \Gamma_{1}$, то $|\widehat{B}(\gamma)(1-g(\gamma))| \leqslant 2|\widehat{B}(\gamma)|<2 \delta n / 2=\delta n$, а при $\gamma \in \Gamma_{1}$ справедливо равенство $|\widehat{B}(\gamma)(1-g(\gamma))|=0$.

2) Пусть $\left|G_{1}\right|<L^{\prime}$. Выберем $d$, так чтобы для $P=\{d i \mid L-1 \leqslant i \leqslant L-1\}$ условия пп. (А) и (В) были выполнены. Отметим, что при $\gamma \in \Gamma \backslash \Gamma_{1}$ п. (В) выполнен. Теперь оценим величину $1-g(\gamma)$. Фиксируем $\gamma \in \Gamma$ и через $\beta$ обозначим $\arg \gamma(d) \in[-\pi, \pi)$. Таким образом,

$$
\begin{gathered}
0 \leqslant 1-g(\gamma)=1-\frac{1}{2 L-1} \sum_{j=-L-1}^{L-1}(\cos j \beta+i \sin j \beta)= \\
=1-\frac{1}{2 L-1}-\frac{2}{2 L-1} \sum_{j=1}^{L-1} \cos j \beta=\frac{2 L-2}{2 L-1}-\frac{2}{2 L-1} \sum_{j=1}^{L-1} \cos j \beta= \\
=\frac{2}{2 L-1} \sum_{j=1}^{L-1}(1-\cos j \beta) \leqslant \frac{1}{2 L-1} \sum_{j=1}^{L-1}(j \beta)^{2}=\frac{L(L-1)}{6} \beta^{2} \leqslant \frac{(L \beta)^{2}}{6} .
\end{gathered}
$$

Заметим, что если $|\arg \gamma(d)| \leqslant L^{-1} \sqrt{6 \delta n /|\widehat{B}(\gamma)|}$ для всех $\gamma \in R$, то п. (В) выполнен. Также отметим, что для выполнения условия $\operatorname{ord}(d) \geqslant 2 L / \varepsilon$ достаточно, чтобы при некотором $\gamma \in \Gamma$ имело место неравенство

$$
0<|\arg \gamma(d)|<2 \pi \cdot \frac{\varepsilon}{2 L}=\frac{\pi \varepsilon}{L} .
$$

Покажем, что существует такое $d \notin G_{1}$, что при всех $\gamma \in R$

$$
|\arg \gamma(d)| \leqslant \frac{1}{L} \min \left(\pi \varepsilon, \sqrt{\frac{6 \delta n}{|\widehat{B}(\gamma)|}}\right) .
$$

Если $d_{1}, d_{2} \in G$ принадлежат различным смежным классам $G$ по $G_{1}$, то есть $d_{1}-d_{2} \notin G_{1}$, то существует такой характер $\gamma \in R$, что $\gamma\left(d_{1}\right) \neq \gamma\left(d_{2}\right)$. Таким образом, 
для существования $d=d_{1}-d_{2}$ с ограничением $|\arg (\gamma(d))|<\eta_{\gamma}$ достаточно, чтобы количество смежных классов по $G_{1}$ превосходило $\prod_{\gamma \in R}\left(1+\left\lfloor 2 \pi / \eta_{\gamma}\right\rfloor\right)$, то есть

$$
\left|G / G_{1}\right|>\prod_{\gamma \in R}\left(1+L \max \left(\frac{2}{\varepsilon}, \sqrt{\frac{2 \pi|\widehat{B}(\gamma)|}{6 \delta n}}\right)\right) .
$$

Заметим, что справедливы неравенства

$$
\begin{gathered}
\prod_{\gamma \in R}\left(1+L \max \left(\frac{2}{\varepsilon}, \sqrt{\frac{2 \pi|\widehat{B}(\gamma)|}{6 \delta n}}\right)\right) \leqslant \prod_{\gamma \in R}\left(1+2 L \max \left(\frac{1}{\varepsilon}, \sqrt{\frac{|\widehat{B}(\gamma)|}{\delta n}}\right)\right) \leqslant \\
\leqslant(4 L)^{|R|} \prod_{\gamma \in R} \max \left(\frac{1}{\varepsilon}, \sqrt{\frac{|\widehat{B}(\gamma)|}{\delta n}}\right) .
\end{gathered}
$$

В силу равенства Парсеваля имеем

$$
\sum_{\gamma \in \Gamma}|\widehat{B}(\gamma)|^{2}=n \sum_{x \in G}|B(x)|^{2}=n|B| \leqslant n^{2} .
$$

Отсюда и из определения множества $R$ следует $|R| \leqslant 4 \delta^{-2}$. Также заметим, что справедливо неравенство $\max (x, y) \leqslant x^{y}$ при $x \geqslant 1$ и $y \geqslant e^{1 / e}$. Значит,

$$
\begin{aligned}
& (4 L)^{|R|} \prod_{\gamma \in R} \max \left(\frac{1}{\varepsilon}, \sqrt{\frac{|\widehat{B}(\gamma)|}{\delta n}}\right) \leqslant(4 L)^{4 \delta^{-2}}\left(\prod_{\gamma \in R} \max \left(\frac{1}{\varepsilon^{4}},\left(\frac{|\widehat{B}(\gamma)|}{\delta n}\right)^{2}\right)\right)^{1 / 4} \leqslant \\
& \leqslant(4 L)^{4 \delta^{-2}}\left(\varepsilon^{-4}\right)^{\left(4 \delta^{2} n^{2}\right)^{-1}} \sum_{\gamma \in \Gamma}|\widehat{B}(\gamma)|^{2} \leqslant(4 L)^{4 \delta^{-2}} \varepsilon^{-\delta^{-2}} \leqslant(4 L / \varepsilon)^{4 \delta^{-2}}<\frac{n}{L^{\prime}} \leqslant\left|G / G_{1}\right| .
\end{aligned}
$$

Таким образом, существование подмножества $P \subseteq G$, удовлетворяющего требованиям пунктов (А) и (В), доказано. Заметим также, что так как по построению $P$ - либо подгруппа, либо прогрессия, симметричная относительно 0, то $g(\gamma)=|P|^{-1} \sum_{p \in P} \gamma(p)$ есть вещественное число из отрезка $[-1,1]$.

Теперь построим множество $A^{\prime}$. Рассмотрим два случая:

1) Если $P$ - подгруппа, то в качестве $A^{\prime}$ возьмем объединение смежных классов $G$ по $P$, содержащих не менее $\varepsilon|P|$ элементов множества $A$. Тогда

$$
\left|A \backslash A^{\prime}\right| \leqslant \varepsilon|P| \cdot \frac{n}{|P|}=\varepsilon n \text {. }
$$

2) Если $P$ - прогрессия с разностью $d$, то расмотрим структуру гранул типа прогрессии с разностью $d$ и в качестве $A^{\prime}$ возьмем объединение прогрессий, содержащих не менее $\varepsilon L / 2$ элементов множества $A$. Заметим, что не более $n L / \operatorname{ord}(d)$ элементов из «остаточных» множеств не входят ни в одну из гранул. Тогда, с учетом того, что $\operatorname{ord}(d) \geqslant 2 L / \varepsilon$, получим

$$
\left|A \backslash A^{\prime}\right| \leqslant \frac{\varepsilon L}{2} \cdot \frac{n}{L}+\frac{n L}{\operatorname{ord}(d)} \leqslant \varepsilon n .
$$


Утверждения (i) и (ii) леммы обоснованы в обоих случаях.

Докажем п. (iii). Рассмотрим функцию $a_{1}(x)=|P|^{-1}|A \cap(P+x)|$. Заметим, что для преобразования Фурье функции $a_{1}(x)$ справедливо соотношение $\widehat{a}_{1}(\gamma)=$ $g(\gamma) \widehat{A}(\gamma)$. Действительно, с учетом того, что $P=-P$, имеем

$$
\begin{aligned}
& \widehat{a}_{1}(\gamma)=\sum_{x \in G} a_{1}(x) \gamma(x)=\frac{1}{|P|} \sum_{x \in G}|A \cap(P+x)| \gamma(x)=\frac{1}{|P|} \sum_{a \in A} \sum_{p \in P} \gamma(a-p)= \\
& =\frac{1}{|P|}\left(\sum_{a \in A} \gamma(a)\right)\left(\sum_{p \in P} \gamma(-p)\right)=\frac{1}{|P|}\left(\sum_{a \in A} \gamma(a)\right)\left(\sum_{p \in P} \gamma(p)\right)=g(\gamma) \widehat{A}(\gamma) .
\end{aligned}
$$

Рассмотрим два случая: $x \in A^{\prime}$ и $x \notin A^{\prime}$.

Пусть $x \in A^{\prime}$. Если $P$ - подгруппа, то $x+P$ содержит не менее $\varepsilon|P|$ элементов множества $A$, а если $P$ - прогрессия, то $x+P$ содержит гранулу, включающую $x$, и поэтому $|(x+P) \cap A| \geqslant \varepsilon|P| / 4$. В обоих случаях $a_{1}(x) \geqslant \varepsilon / 4=\varepsilon A^{\prime}(x) / 4$.

Если $x \notin A^{\prime}$, то $a_{1}(x) \geqslant 0=\varepsilon A^{\prime}(x) / 4$.

Таким образом, с учетом того, что $|\widehat{A}(\gamma)| \leqslant \sum_{x \in A}|\gamma(x)|=|A| \leqslant n$ и $A \in S F_{k, l}(G)$, имеем

$$
\begin{aligned}
& \text { \# }\left\{\text { решений уравнения (1) в } A^{\prime}\right\}= \\
& =\sum_{\substack{x_{1}+\ldots+x_{k}=\\
=y_{1}+\ldots+y_{l}}} A^{\prime}\left(x_{1}\right) \cdot \ldots \cdot A^{\prime}\left(x_{k}\right) \cdot A^{\prime}\left(y_{1}\right) \cdot \ldots \cdot A^{\prime}\left(y_{l}\right) \leqslant \\
& \leqslant \frac{4^{k+l}}{\varepsilon^{k+l}} \cdot \sum_{\substack{x_{1}+\ldots+x_{k}=\\
=y_{1}+\ldots+y_{l}}} a_{1}\left(x_{1}\right) \cdot \ldots \cdot a_{1}\left(x_{k}\right) \cdot a_{1}\left(y_{1}\right) \cdot \ldots \cdot a_{1}\left(y_{l}\right)= \\
& =\frac{4^{k+l}}{\varepsilon^{k+l}} \cdot \sum_{\substack{x_{1}+\ldots+x_{k}=\\
=y_{1}+\ldots+y_{l}}}\left(a_{1}\left(x_{1}\right) \cdot \ldots \cdot a_{1}\left(x_{k}\right) \cdot a_{1}\left(y_{1}\right) \cdot \ldots \cdot a_{1}\left(y_{l}\right)-\right. \\
& \left.-A\left(x_{1}\right) \cdot \ldots \cdot A\left(x_{k}\right) \cdot A\left(y_{1}\right) \cdot \ldots \cdot A\left(y_{l}\right)\right)= \\
& =\frac{4^{k+l}}{\varepsilon^{k+l}} \cdot \frac{1}{n} \cdot \sum_{\gamma \in \Gamma}\left(\left|\widehat{a}_{1}(\gamma)\right|^{2 l}\left(\widehat{a}_{1}(\gamma)\right)^{k-l}-|\widehat{A}(\gamma)|^{2 l}(\widehat{A}(\gamma))^{k-l}\right)= \\
& =\frac{4^{k+l}}{\varepsilon^{k+l}} \cdot \frac{1}{n} \cdot \sum_{\gamma \in \Gamma}|\widehat{A}(\gamma)|^{2 l}(\widehat{A}(\gamma))^{k-l}\left(|g(\gamma)|^{2 l}(g(\gamma))^{k-l}-1\right) \leqslant \\
& \leqslant \frac{4^{k+l}}{\varepsilon^{k+l}} \cdot \frac{1}{n} \cdot \sum_{\gamma \in \Gamma}|\widehat{A}(\gamma)|^{k+l}\left|1-(g(\gamma))^{k+l}\right| \leqslant \\
& \leqslant \frac{4^{k+l}}{\varepsilon^{k+l}} \cdot \frac{1}{n} \cdot(k+l) \cdot \max _{\gamma \in \Gamma}|\widehat{A}(\gamma)|^{k+l-3} \cdot \max _{\gamma \in \Gamma}|\widehat{A}(\gamma)||1-g(\gamma)| \cdot \sum_{\gamma \in \Gamma}|\widehat{A}(\gamma)|^{2} \leqslant \\
& \leqslant \frac{4^{k+l}}{\varepsilon^{k+l}} \cdot \frac{1}{n} \cdot(k+l) \cdot n^{k+l-3} \cdot \max _{\gamma \in \Gamma}|\widehat{A}(\gamma)||1-g(\gamma)| \cdot n|A| \leqslant \\
& \leqslant \frac{4^{k+l}}{\varepsilon^{k+l}} \cdot n^{k+l-4} \cdot(k+l) \cdot \delta n \cdot n^{2}=\varepsilon n^{k+l-1} .
\end{aligned}
$$

Лемма 4 доказана.

4 Дискретная математика, т. 26 № 4 
В следующей теореме доказывается существование семейства гранул.

Теорема 6. Пусть $G$ - абелева группа порядка $n$ и $n$ достаточно велико. Тогда существует семейство $\mathcal{F}$ подмножеств группы $G$, удовлетворяющее условиям

(i) $\log |\mathcal{F}| \leqslant 2 n(k+l-1)^{-1 / 2}(\log n)^{-(4(k+l)+6)^{-1}}$;

(ii) для каждого $A \in S F_{k, l}(G)$ существует $F \in \mathcal{F}$ такое, что $A \subseteq F$;

(iii) всякое $F \in \mathcal{F}$ содержит не более $n^{k+l-1}(\log n)^{-(2(k+l)+3)^{-1}}$ решений уравнения (1).

Доказательство. Положим $L=L^{\prime}=\lfloor\log n\rfloor$ и $\varepsilon=(k+l+1)^{-1}(\log n)^{-(2(k+l)+3)^{-1}}$. Заметим, что при достаточно большом $n$ такой выбор параметров удовлетворяет условию леммы 4. Таким образом, для каждого множества $A \in S F_{k, l}(G)$, применяя лемму 4 , построим множество $A^{\prime}$. Положим $\mathcal{F}=\left\{A \cup A^{\prime} \mid A \in S F_{k, l}(G)\right\}$. Тогда п. (ii) выполнен автоматически.

Отсюда и из п. (ii) леммы 4 следует, что мощность семейства $\mathcal{F}$ не превосходит количества множеств, являющихся объединением $L$-гранулы с некоторым подмножеством группы $G$ мощности не более $\varepsilon n$. Таким образом, из лемм 2 и 3 вытекает, что $\log |\mathcal{F}| \leqslant 3 n / L+n \sqrt{\varepsilon}$, что при достаточно большом $n$ не превосходит $2 n \sqrt{\varepsilon}$.

Заметим также, что при добавлении элемента в множество в нем может образоваться не более $(k+l) n^{k+l-2}$ новых решений уравнения (1). Из этого следует, что в каждом множестве $F \in \mathcal{F}$ не более $\varepsilon n^{k+l-1}+\varepsilon(k+l) n^{k+l-1}=$ $n^{k+l-1}(\log n)^{-(2(k+l)+3)^{-1}}$ решений уравнения (1).

Теорема 6 доказана.

\section{4. Доказательство теоремы 4}

Пусть $G$ - абелева группа порядка $n$. По теореме 6 существует семейство $\mathcal{F}$ подмножеств (гранул) группы $G$, удовлетворяющее условиям (i)-(iii). Пусть $F \in \mathcal{F}$. Фиксируя $F$ и применяя теорему 5 при $A_{1}=\ldots=A_{k}=F$ и $A_{k+1}=\ldots=A_{k+l}=-F$, получим, что существует такое $F^{\prime} \subseteq F$, что $\left|F \backslash F^{\prime}\right|=\bar{o}(n)$ и $F^{\prime} \in S F_{k, l}(G)$. Отсюда следует, что $|F| \leqslant \mu_{k, l}(G)+\bar{o}(n)$, где $\mu_{k, l}(G)$ - максимальная мощность множества из $S F_{k, l}(G)$. Из того, что $\log |\mathcal{F}|=\bar{o}(n)$ (пункт (i) теоремы 6) получим, что количество подмножеств всех множеств семейства $\mathcal{F}$, не превосходит $2^{\mu_{k, l}(G)+\bar{o}(n)}$.

В силу п. (ii) теоремы 6 всякое множество из $S F_{k, l}(G)$ является подмножеством некоторого множества из семейства $\mathcal{F}$. Отсюда следует, что

$$
\log \left|S F_{k, l}(G)\right|=\mu_{k, l}(G)+\bar{o}(n) .
$$

Теорема 4 доказана.

Автор выражает признательность профессору А.А. Сапоженко за постановку задачи и внимание к этой работе.

\section{Список литературы}

1. Cameron P. J., Erdös P., "On the number of sets of integers with various properties", J. Number Theory, 1990, 61-79.

2. Calkin N. J., "On the number of sum-free set", Bull. London Math. Soc., 22 (1990), 140-144.

3. Alon N., "Independent sets in regular graphs and sum-free subsets of Abelian groups", Israel J. Math., 73 (1991), 247-256. 
4. Сапоженко А. А., "Гипотеза Камерона-Эрдёша", Докл. РАН, 393:6 (2003), 749-752.

5. Green B., "The Cameron-Erdös conjecture.", Bull. London Math. Soc., 36:6 (2004), 769-778.

6. Сапоженко А. А., "О числе множеств, свободных от сумм, в абелевых группах", Вестник МГУ, сер. 1. Матем., мех., 4 (2002), 14-17.

7. Lev V.F., Luczak T., Schoen T., "Sum-free sets in Abelian groups", Israel J. Math., 125 (2001), 347-367.

8. Lev V.F., Schoen T., "Cameron-Erdös modulo a prime", Finite Fields Appl., 8:1 (2002), 108-119.

9. Green B., Ruzsa I., "Sum-free sets in Abelian groups", Israel J. Math., 147 (2005), 157-188.

10. Сапоженко А. А., "Решение проблемы Камерона-Эрдёша для групп простого порядка", Вычисл. мат. и матем. физика, 49:8 (2009), 1-7.

11. Calkin N. J., Taylor A.C., "Counting sets of integers, no $k$ of which sum to another", J. Number Theory, 57 (1996), 323-327.

12. Bilu Yu., "Sum-free sets and related sets", Combinatorica, 18:4 (1998), 449-459.

13. Calkin N. J., Thomson J. M., "Counting generalized sum-free sets", J. Number Theory, 68 (1998), 151-160.

14. Schoen T., "A note on the number of $(k, l)$-sum-free sets", Electronic J. Combinatorics, 7:1 (2000), 1-8.

15. Саргсян В.Г., “44-51”, Вестник МГУ, сер. 15. Вычисл. матем. и киберн., 2 (2012).

16. Lev V.F., "1-25", J. fur die reine und angew. Math., 555 (2003).

17. Green B., "A Szemerédi-type regularity lemma in Abelian groups", Geom. and Funct. Anal, 15:2 (2005), 340-376.

18. Bajnok B., "On the maximum size of a $(k, l)$-sum-free subset of an Abelian group", J. Number Theory, 5:6 (2009), 953-971.

19. Саргсян В. Г., "О максимальной мощности множества, $k$-свободного от нуля, в абелевой группе", Дискр. анализ и исслед. операций, 20:3 (2013), 45-64.

Статья поступила 20.03.2014. 